\title{
Cardiotoxicity in breast cancer treatment: What about left ventricular diastolic function and left atrial function?
}

\author{
Ana Teresa Timóteo $\mathrm{PhD}^{1}$ (D) | Luisa Moura Branco MD ${ }^{1}$ | Frederico Filipe $\mathrm{MD}^{2}$ | \\ Ana Galrinho MD ${ }^{1}$ | Pedro Rio MD ${ }^{1}$ | Guilherme Portugal $M^{1}$ | Sónia Oliveira $M^{2}$ | \\ Rui Cruz Ferreira MD ${ }^{1}$
}

${ }^{1}$ Cardiology Department, Santa Marta Hospital, Centro Hospitalar Universitário Lisboa Central, Lisbon, Portugal

${ }^{2}$ Oncology Department, Santo António Capuchos Hospital, Centro Hospitalar Universitário Lisboa Central, Lisbon, Portugal

\section{Correspondence}

Ana Teresa Timóteo, Cardiology

Department, Santa Marta Hospital, Centro Hospitalar Universitário Lisboa Central, Rua Santa Marta, 1169-024 Lisboa, Portugal.

Email: ana_timoteo@yahoo.com

\begin{abstract}
Aims: Cardiotoxicity is a possible complication of cancer treatment, particularly with anthracyclines and anti-HER2 drugs. Systolic dysfunction has already been described. Diastolic dysfunction and left atrial function are less studied. We sought to analyze the impact of cardiotoxic treatments on left ventricular diastolic function and left atrial (LA) function.

Methods and Results: Retrospective study of 100 patients (all women, with a mean age of $54 \pm 12$ years) with three exams in the span of 1 year during treatment for breast cancer. Patients with previous cancer treatment, coronary artery disease, significant valvular disease, and atrial arrhythmias were excluded. Diastolic dysfunction was classified according to international guidelines and left atrial strain was analyzed by two-dimensional speckle tracking. In our sample, $74 \%$ received anthracyclines, $83 \%$ anti-HER2, and $76 \%$ radiation treatment. In the follow-up, $20 \%$ developed new or worsening diastolic dysfunction. Age was the only independent predictor (OR $1.93,95 \% \mathrm{Cl} 1.04-3.58, P=.037)$. In left atrial function, only the contractile function was significantly reduced in $20.8 \%$ of the patients and age was also the only independent predictor, but with a protective effect (OR 0.51, 95\% Cl 0.28-0.91, $P=.023$ ). Conclusions: During breast cancer treatment, $20 \%$ of the patients develop new or worsening diastolic dysfunction, being age the main determinant, suggesting higher impact of chemotherapy in older patients. Contractile left atrial function is also compromised but, in this case, age seems to be protective. Our results support a stricter surveillance in older patients together to eventually adjust chemotherapy regimens.
\end{abstract}

KEYWORDS

breast cancer, chemotherapy, diastolic function, left atrial strain

\section{1 | INTRODUCTION}

Breast cancer is one of the most common cancers worldwide. In 2018, there were more than two million new cases, representing $11.6 \%$ of all new cancer cases. ${ }^{1}$ In terms of cause of death, it represents $6.6 \%$ of all deaths from cancer. ${ }^{1}$ From 2006 to 2016 , the number of deaths from breast cancer increased by $17 \%$, but age-standardized mortality rates decreased by $9.9 \%{ }^{2}$ There was also a significant reduction of $12.4 \%$ in the mean age-standardized years of life lost rate in countries with high socio-demographic index. ${ }^{2}$ Portuguese data showed that in 2015, there were 1683 deaths due to breast cancer with an adjusted mortality rate of 19.3 (per 100 000). ${ }^{3}$ Treatment efficacy has been 
increasing, and patient's survival and longevity have also improved. Cardiotoxicity is very common in breast cancer treatment, both early and late after treatment, and thus, cardiac surveillance is highly recommended in these patients. ${ }^{4,5}$ Speckle-tracking echocardiography (STE) has been validated as a quantitative assessment tool for left ventricular (LV) systolic function in this setting and is currently recommended in the surveillance of these patients during and after cancer treatment. ${ }^{4}$ Worsening of LV myocardial deformation is common in breast cancer patients undergoing chemotherapy, and an impaired Global Longitudinal Strain (GLS) in patients with preserved LV ejection fraction is independently associated with increased incidence of chemotherapy-induced cardiotoxicity and is thus an earlier predictor compared to the usual measurement of LV ejection fraction., ${ }^{4,6}$

Diastolic dysfunction is common in patients with cancer, both at baseline and during treatment, mainly related to the age group usually affected with cancer ${ }^{4}$; however, no evidence has shown that treatment should be stopped based on these findings. ${ }^{4}$ In fact, diastolic parameters have not yet demonstrated value in predicting subsequent cardiotoxicity.

More recently, GLS by STE has been described for assessment of regional and global left atrial (LA) function. ${ }^{7}$ Atrial strain has been evaluated in multiple conditions, such as hypertension, diabetes, heart failure, ischemic and valvular heart disease, and atrial fibrillation, including for assessment of prognostic implications. ${ }^{8-14}$

It was our objective to evaluate in patients submitted to breast cancer treatment, the effect of treatment on LV diastolic function, its prognostic effect in subsequent LV systolic function, and the effects in LA function as assessed by STE.

\section{2 | METHODS}

\section{1 | Population}

Retrospective analysis of all consecutive patients prospectively included in a single-center echocardiographic registry of patients submitted to cardiotoxic agents in the setting of Oncology. Patient inclusion started in 2014, and all patients that performed at least three echocardiograms in the span of 1 year in our laboratory during chemotherapy for breast cancer were included in the present analysis. Patients were excluded if they had a previous cancer treatment (with chemotherapy or radiotherapy), or in case, the treatment was started before the baseline echocardiographic assessment. A previous myocardial infarction, coronary revascularization, significant valvular heart disease, and atrial fibrillation/flutter or pacemaker were also exclusion criteria.

Hypertension was defined by a previous diagnosis or treatment with anti-hypertensive drugs. Diabetes was defined by a previous diagnosis or treatment with anti-diabetic drugs. Obesity was defined by a body mass index above $30 \mathrm{Kg} / \mathrm{m}^{2}$.

\section{2 | Echocardiography}

A complete standard echocardiographic study was performed using commercially available systems (Vivid $7^{\mathrm{TM}}$, Vivid $9^{\mathrm{TM}}$, and VividE95 $5^{\mathrm{TM}}$;
GE Healthcare). The patient was positioned in left lateral position, and the study was performed with a $3.5 \mathrm{MHz}$ transducer. Left ventricular ejection fraction was assessed with the biplane method of disks (modified Simpson's rule). ${ }^{15}$ The following four variables were evaluated for identifying LV diastolic dysfunction: mitral annular $\mathrm{e}^{\prime}$ velocity (septal and lateral), average $\mathrm{E} / \mathrm{e}^{\prime}$ ratio, LA maximum volume index, and tricuspid regurgitation peak velocity. ${ }^{16}$ LA volume was calculated with the biplane algorithm, which includes the 4-chamber and 2-chamber apical views. ${ }^{15}$ Diastolic dysfunction was classified according to the European Association of Cardiovascular Imaging (EACVI) guidelines. ${ }^{16}$ Left ventricular GLS was analyzed by two-dimensional STE. Images were acquired in the three standard apical views, and the transducer settings of the B-mode image were adjusted to achieve a frame rate of at least 55 frames per second (fps) (preferably set at 60-80 fps). Optimization of endocardial and myocardial definition was obtained by adjusting the gray scale. Images were digitally stored in cineloop format that included three sequential beats and were transferred to a workstation for subsequent offline analysis using the software package EchoPAC ${ }^{T m}$ (version 202, GE Healthcare). The borders of the LV endocardium were manually traced, and additional lines were automatically generated by the program near the epicardium. Speckles were traced during the cardiac cycle, in each frame. Whenever necessary, the region of interest was adjusted manually by the operator. Left ventricular GLS was the average of all 18 segments. Left atrial strain was analyzed offline with the same software and with Q-analysis. Thus, dedicated software for atrial analysis was not used. As recommended, LA longitudinal strain was obtained from an optimized apical 4-chamber view of the LA. ${ }^{7}$ The 4-chamber view was optimized in terms of orientation, depth, and gain, avoiding LA foreshortening and allowing visualization of the entire LA throughout the cardiac cycle. The region of interest size and shape was adjusted in order to include the thickness of LA wall. LA delineation was performed in a similar manner as for the LV, and it was manually edited if needed. Endocardial contour was extrapolated across the orifice of pulmonary veins and LA appendage. Reference was placed at the onset of the R-wave (R-R gating). With this specific gating, all strains are positive (Figure 1). There are two peaks that correspond to reservoir function (first peak-eR) and atrial contractile function (second peak $-\varepsilon C T$ ). The difference between reservoir strain and atrial contractile strain values reflect conduit function $(\varepsilon C D)$. Strain measurements were made by a single operator. Because LA strain analysis is not performed with specific software, additional reproducibility analysis was performed.

Cancer treatment-related cardiac dysfunction (cardiotoxicity) was defined by a reduction of $10 \%$ points in LV ejection fraction to a value below $50 \%$ (lower limit of normal) or by a relative percentage reduction of more than $15 \%$ of LV GLS from baseline. ${ }^{4}$ Because there are no cutoffs for LA cardiotoxicity, we considered significant a reduction in the LA functional strain parameters above $15 \%$ to a value below the 10th percentile (calculated in the baseline evaluation) for each atrial function strain parameter.

All patients gave their informed consent to be included in the registry, and the study complies with the Helsinki declaration. 


\section{3 | Statistical analysis}

Assuming that $5 \%$ and $10 \%$ of the pairs have diastolic dysfunction in the first and second observation, respectively, that the correlation between paired observations is at least $70 \%$, and applying continuity correction, the study would require a sample size of 68 pairs to achieve a power of $80 \%$ and a two-sided significance of $5 \%$ to detect a difference of 0.10 between marginal populations. For that reason, 100 patients are our ideal sample size. For LA strain analysis, with a smaller sample of 75 individuals (assuming that in $25 \%$ of the patients it would not be possible to evaluate LA strain), with the same power and level of significance, assuming a correlation of 0.85 for LA strain parameters, we obtain an effect size of 1.796 .

Continuous variables are expressed as mean and standard deviation. Normality and homogeneity of variance were tested with Kolmogorov-Smirnov's test and Levene's test, respectively. Categorical variables are reported as absolute frequencies and percentages. Differences between groups for categorical variables were tested with the chi-square test or Fisher's exact test, as appropriate. For continuous variables, paired Student's $t$ test was used.

Multivariate logistic regression models were used to assess the factors associated with LV diastolic dysfunction and LA dysfunction. Factors that remained significant at the 0.10 level in univariate analysis were considered to be significant contributors and were included in the final models. Multicollinearity was also assessed and when identified, those variables were removed from the final multivariate model. Estimates of the association between predictors and endpoints are presented as odds ratio (OR) with $95 \%$ confidence interval (Cl). The model's calibration was assessed with the HosmerLemeshow test.

To establish and quantify reproducibility of LA strain analysis, agreement and intra- and inter-observer reproducibility were assessed using the interclass correlation coefficient and reliability coefficients (Cronbach's alpha) with two-way mixed effects models.
Mean differences and limits of agreement were analyzed with BlandAltman plots. Twenty-five consecutive patients were selected for reproducibility analysis, and the two operators were blinded for patient status and previous results.

IBM SPSS Statistics Software, version 19.0.0.2 (IBM SPSS Inc) was used for all statistical analysis. All statistical tests were twosided with a critical value of 0.05 for statistical significance.

\section{3 | RESULTS}

\section{1 | Population}

From an initial sample of 111 patients, 11 were excluded due to previous cancer treatment and the final sample included 100 patients, all females, with a mean age of $54 \pm 12$ years ( $28-83$ years). The time interval between the first and second exam was $5.7 \pm 1.8$ months, and the third exam was performed $11.5 \pm 2.3$ months after the baseline. Baseline characteristics and cancer treatment are detailed in Table 1. All patients had a baseline LV ejection fraction $>50 \%$ and LV GLS > -18.5\%. During treatment, three patients developed LV systolic dysfunction as assessed by LV ejection fraction and 24 patients by LV GLS. Regarding treatment, the most commonly used chemotherapy regimens were as follows: FEC $\times 3$ (5-fluorouracil, Epirubicin, Cyclophosphamide, with a maximum total dose of $300 \mathrm{mg} /$ $\mathrm{m}^{2}$ of epirubicin) followed by DTP $\times 3$ (Doctaxel, Traztuzumab, Pertuzumab); AC $\times 4$ (Doxorubicin, Cyclophosphamide, with a maximum total dose of $240 \mathrm{mg} / \mathrm{m}^{2}$ of doxorubicin) followed by DT $\times 4$ (Docetaxel + Trastuzumab); TCH $\times 6$ (Docetaxel, Carboplatin, Trastuzumab). In $81 \%$ of cases, these regimens were followed by 1 year of trastuzumab alone every 21 days in the dose of $6 \mathrm{mg} / \mathrm{Kg}$. Radiation treatment occurred at a median of 5 months after the baseline echocardiogram, and for that reason, the implications in LV diastolic function and LA function in the second echocardiographic evaluation were not analyzed.

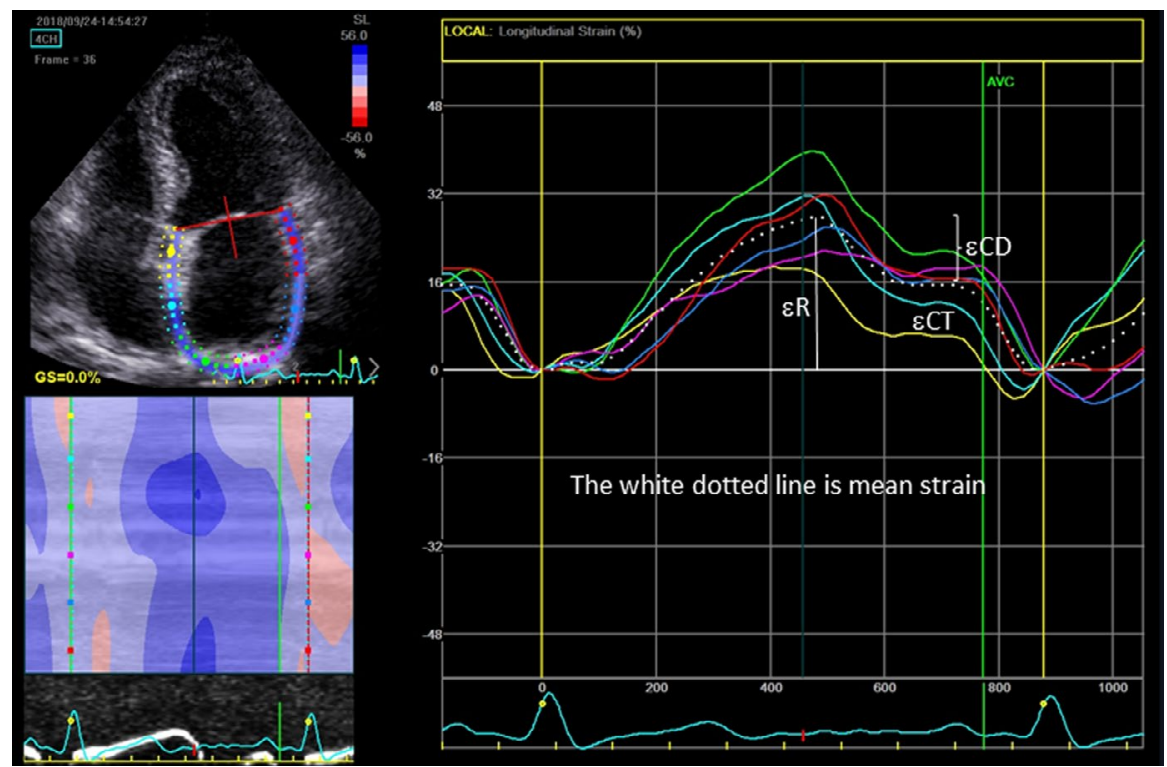

FIGURE 1 Left atrial strain analysis by speckle-tracking echocardiography. $\varepsilon C D=$ conduit strain; $\varepsilon C T=$ contractile strain; $\varepsilon \mathrm{R}=$ reservoir strain 
TAB LE 1 Baseline characteristics and cancer treatment during the study

\begin{tabular}{|lr|}
\hline Cardiovascular risk factors $\mathbf{n}(\%)$ & \\
\hline Hypertension & $33(33.0)$ \\
\hline Obesity & $30(30.0)$ \\
\hline Diabetes & $12(12.0)$ \\
\hline Chemotherapy (\%) & \\
\hline Anthracylines & $74(74.0)$ \\
\hline Doxorubicin & $53(53.0)$ \\
\hline Epirubicin & $21(21.0)$ \\
\hline Cyclophosphamide & $78(78.0)$ \\
\hline Docetaxel & $80(80.0)$ \\
\hline Paclitaxel & $11(11.0)$ \\
\hline Anti-HER2 & $83(83.0)$ \\
\hline Trastuzumab & $81(81.0)$ \\
\hline Pertuzumab & $17(17.0)$ \\
\hline Aromatase inhibitors & $23(23.0)$ \\
\hline 5-Fluorouracil & $24(24.0)$ \\
\hline Metotrexate & $3(3.0)$ \\
\hline Carboplatin & $9(9.0)$ \\
\hline Goserelin & $7(7.0)$ \\
\hline Tamoxifen & $49(49.0)$ \\
\hline Anthracyclines + Anti-HER2 & $56(56.0)$ \\
\hline Trastuzumab + Pertuzumab & $17(17.0)$ \\
\hline Neoadjuvant chemotherapy (\%) & $32(32.0)$ \\
\hline Radiation treatment (\%) & $76(76.0)$ \\
\hline
\end{tabular}

Abbreviations: HER = human epidermal growth factor receptor.

\section{2 | Left ventricular diastolic function}

During follow-up, there was a significant increase in LA volume (Table 2). In regard to diastolic function, there was a steady worsening from the baseline to the third echo examination (Table 2). In total, 20 patients developed new or worsening diastolic dysfunction during treatment. In an exploratory analysis, the main potential predictors were age, hypertension, docetaxel use, and aromatase inhibitors use (Table 3). However, in multivariate analysis, only age remained as an independent predictor of diastolic dysfunction development. Neither anthracyclines use (OR 0.78, 95\% Cl 0.26-2.29), Anti-HER2 use (OR 0.45, 95\% Cl 0.15-1.40), nor both (OR 0.44, 95\% Cl 0.161.21), nor radiation therapy (OR $1.44,95 \% \mathrm{Cl} 0.38-5.57-$ comparison only for the third exam) nor LV systolic dysfunction development (OR 1.20 , 95\% Cl 0.41-3.54) was associated with LV diastolic dysfunction development/worsening during chemotherapy treatment.

In patients with baseline diastolic dysfunction, the risk of development of systolic cardiotoxicity during the 12 months follow-up was not significant (OR 2.03, 95\% Cl 0.39-10.66). Patients with normal LV systolic function in the second exam but that developed new or worsening diastolic dysfunction did not have an increased risk of cardiotoxicity in the third exam (OR 2.31, 95\% Cl 0.51-10.45).
There were no heart failure hospitalizations. In $37 \%$ of the patients, mild fatigue was reported and mild pedal edema in $7 \%$. However, fatigue is a commonly reported side effect of chemotherapy and in most cases unrelated to heart failure. There was no association between fatigue and left ventricular diastolic dysfunction (only $35 \%$ of the patients with fatigue had diastolic dysfunction, $P=.836$ ), and the same was observed for peripheral edema (10\% of the patients with edema had diastolic dysfunction, $P=.625)$. There was, however, some association, as expected, with left ventricular systolic dysfunction $(100 \%$ of patients with systolic dysfunction had fatigue, $P=.048 ; 33 \%$ of the patients with systolic dysfunction had edema, $P=.197$ ).

\section{3 | Left atrial function assessment}

Reproducibility analysis showed excellent reproducibility both for intra- and inter-observer, despite the use of a nondedicated software for LA strain analysis (Table S1). It was possible to assess LA strain in 77 patients ( $53 \pm 12$ years). Risk factors and treatment proportions are similar to the main study group, as well as the time interval between studies. In this sample, it was also observed an increase in LA volume and LV systolic dysfunction during follow-up (Table 4). However, we did not observe any significant worsening of mean left atrial function as assessed by strain analysis. The selected cutoff for $\varepsilon R$ was $16.8 \%, 8.0 \%$ for $\varepsilon \mathrm{CT}$, and $7.1 \%$ for $\varepsilon \mathrm{CD}$. The parameter that had more important worsening during treatment was $\varepsilon \mathrm{CT}$ (Table 5). No factor was independently associated with $\varepsilon \mathrm{R}$ or $\varepsilon \mathrm{CD}$ reduction (Table 6). For $\varepsilon \mathrm{CT}$, it was only age that showed an inverse relationship. There was also a trend to be independently associated with baseline LV ejection fraction.

\section{4 | DISCUSSION}

During the first year of breast cancer treatment, there is a significant worsening in diastolic function, being age the only independent predictor. Neither baseline diastolic dysfunction nor LV systolic dysfunction had any significant impact on LV diastolic dysfunction. Regarding LA function, the contractile function was the one that was more frequently compromised, being age a protective factor.

In breast cancer treatment, most regimens include the use of anthracyclines, taxanes, and inhibitors of human epidermal growth factor receptor 2 (anti-HER2) drugs, which are associated with LV dysfunction. ${ }^{4}$ Anthracyclines can induce early cardiotoxicity due to cell necrosis (type I toxicity) and consequent myocardial dysfunction. ${ }^{5}$ It causes irreversible cardiac damage and progressive cardiac remodeling as a late consequence. ${ }^{4,5}$ The most common hypothesis is the generation of reactive oxygen species, and lipid peroxidation of the cell membrane that damages the cardiomyocyte. ${ }^{4,5}$ These agents have a cumulative dose relationship with cardiotoxicity. When a cumulative lifetime dose of doxorubicin (or equivalent) exceeds $400 \mathrm{mg} / \mathrm{m}^{2}$, the incidence of heart failure is up to $5 \% .{ }^{4,5}$ However, there is considerable variability among patients in their susceptibility. ${ }^{4}$ Early effects occur within the first year of treatment in about $98 \%$ of the cases. ${ }^{4}$ Currently, the doses in routine clinical 
TAB LE 2 Echocardiographic results for diastolic function

\begin{tabular}{|c|c|c|c|c|c|c|}
\hline Variable & Echo 1 & Echo 2 & $P$-value & Echo 3 & $P$-value (2 vs 3 ) & $P$-value (1 vs 3 ) \\
\hline Mitral A (cm/s) & $75 \pm 19$ & $76 \pm 18$ & .691 & $74 \pm 18$ & .292 & .565 \\
\hline Mitral DecT (m/s) & $209 \pm 52$ & $191 \pm 40$ & .060 & $191 \pm 54$ & .996 & .018 \\
\hline Mitral E's (cm/s) & $8.2 \pm 2.6$ & $8.1 \pm 2.4$ & .822 & $8.1 \pm 2.4$ & 1.000 & .568 \\
\hline Mitral $E^{\prime}$ mean $(\mathrm{cm} / \mathrm{s})$ & $9.9 \pm 2.6$ & $9.7 \pm 2.7$ & .441 & $9.6 \pm 2.5$ & .509 & .290 \\
\hline$E / E^{\prime}$ & $8.1 \pm 2.4$ & $8.5 \pm 2.3$ & .177 & $8.3 \pm 2.6$ & .651 & .311 \\
\hline TR vel (m/s) & $2.3 \pm 0.3$ & $2.4 \pm 0.3$ & .490 & $2.4 \pm 0.3$ & .097 & .454 \\
\hline $\mathrm{LA} / \mathrm{BSA}\left(\mathrm{mL} / \mathrm{m}^{2}\right)$ & $24 \pm 7$ & $25 \pm 7$ & .115 & $27 \pm 9$ & .100 & .003 \\
\hline Normal & 94 & 88 & & 84 & & \\
\hline Grade 1 & 6 & 12 & & 11 & & \\
\hline Grade 2 & 0 & 0 & & 5 & & \\
\hline
\end{tabular}

Abbreviations: BSA = body surface area; DecT = deceleration time; LA = left atrial; LVEF = left ventricular ejection fraction; LVGLS = left ventricular global longitudinal strain; TR = tricuspid regurgitation.

\begin{tabular}{|c|c|c|c|c|}
\hline & Univariate & & Multivariate & \\
\hline Variable & Odds ratio $(95 \% \mathrm{Cl})$ & $P$-value & Odds ratio $(95 \% \mathrm{Cl})$ & $P$-value \\
\hline $\begin{array}{l}\text { Age (per 10-y } \\
\text { increase) }\end{array}$ & $2.79(1.65-4.71)$ & $<.001$ & $1.93(1.04-3.58)$ & .037 \\
\hline Hypertension & $7.49(2.53-22.2)$ & $<.001$ & $2.74(0.74-10.19)$ & .132 \\
\hline Docetaxel & $0.36(0.12-1.08)$ & .068 & $0.49(0.13-1.89)$ & .301 \\
\hline Aromatase inhibitor & $3.86(1.35-11.06)$ & .012 & $2.04(0.59-7.12)$ & .262 \\
\hline
\end{tabular}

TAB LE 3 Logistic regression analysis for predictors of left ventricular diastolic dysfunction development use were reduced, and typical cumulative exposure is now mostly in the $240-360 \mathrm{mg} / \mathrm{m}^{2}$ range for doxorubicin and $450-600 \mathrm{mg} / \mathrm{m}^{2}$ for epirubicin. ${ }^{5}$ Cyclophosphamide cardiotoxicity is relatively rare, and it occurs within days of drug administration, with the same risk factors as for anthracyclines. ${ }^{4}$ Docetaxel, used frequently in combination with or after anthracyclines, cyclophosphamide or trastuzumab, also increases the incidence of heart failure. ${ }^{4}$ Taxanes also reduce doxorubicin elimination, resulting in higher plasmatic levels. ${ }^{4}$ In this regard, paclitaxel is more cardiotoxic that docetaxel. Anti-HER2 drugs, such as trastuzumab and pertuzumab, can be given concomitantly with anthracyclines, but due to the high incidence of cardiotoxicity, it is more commonly used after anthracyclines or using an anthracycline-free chemotherapy regimen with antimetabolites or alkylating agents. ${ }^{4}$ In contrast to other agents, trastuzumab toxicity manifests during treatment and unlike anthracyclines, long-term studies are reassuring in terms of absence of late effects and it is not cumulative dose-related. ${ }^{4} \mathrm{~A}$ drug-free interval between the two agents is recommended. ${ }^{4,5}$ With this drug, cardiac dysfunction appears to rise from impairment of contractility due to cell dysfunction (type II toxicity) rather than loss of myocytes and is potentially reversible. ${ }^{5}$

Regarding radiotherapy, there is a longtime delay between exposure and clinical manifestation of radiation-induced cardiotoxicity and it results from marked interstitial myocardial fibrosis. ${ }^{4}$ Also, continuous improvements in radiation techniques are reducing its incidence. ${ }^{4}$ In general, the main risk factors for cardiotoxicity are the cumulative dose of some drugs (such as anthracyclines), age above 65 years, preexisting cardiac diseases leading to increased wall stress, arterial hypertension, concomitant use of mediastinal radiation and/or a concomitant or incorrect timing of administration of multiple cancer drugs (alkylating, antimicrotubule agents, and particularly immunotherapy and targeted therapies) with potential for cardiotoxic effects from interactions among the different therapeutic modalities. ${ }^{4,5}$

If all these drugs have an effect on LV myocyte, they can also induce diastolic dysfunction (that can potentially be used as an earlier indicator of progressive LV systolic dysfunction), or LA dysfunction. However, diastolic parameters have not yet demonstrated value in predicting subsequent chemotherapy-related cardiotoxicity. E/e' ratio 
TAB LE 4 Echocardiographic results for the left atrial analysis

\begin{tabular}{|c|c|c|c|c|c|c|}
\hline Variable & Echo 1 & Echo 2 & $P$-value & Echo 3 & $P$-value (2 vs 3 ) & $P$-value (1 vs 3 ) \\
\hline Mitral A (cm/s) & $75 \pm 19$ & $74 \pm 17$ & .617 & $73 \pm 18$ & .584 & .271 \\
\hline Mitral DecT (m/s) & $208 \pm 48$ & $192 \pm 42$ & .026 & $193 \pm 58$ & .898 & .082 \\
\hline Mitral E's (cm/s) & $8.1 \pm 2.7$ & $8.2 \pm 2.4$ & .629 & $8.0 \pm 2.4$ & .281 & .464 \\
\hline Mitral $E^{\prime}$ mean $(\mathrm{cm} / \mathrm{s})$ & $9.9 \pm 2.6$ & $9.8 \pm 2.6$ & .882 & $9.6 \pm 2.5$ & .199 & .227 \\
\hline$E / E^{\prime}$ & $8.1 \pm 2.4$ & $8.3 \pm 2.3$ & .425 & $8.3 \pm 2.7$ & .866 & .473 \\
\hline TR vel $(\mathrm{m} / \mathrm{s})$ & $2.3 \pm 0.3$ & $2.3 \pm 0.3$ & .716 & $2.4 \pm 0.3$ & .432 & .730 \\
\hline LA/BSA $\left(\mathrm{mL} / \mathrm{m}^{2}\right)$ & $24 \pm 7$ & $25 \pm 7$ & .563 & $27 \pm 9$ & .044 & .014 \\
\hline LVEF (\%) & $64 \pm 7$ & $62 \pm 7$ & .011 & $60 \pm 7$ & .139 & $<.001$ \\
\hline Normal & $72(93.5)$ & $71(92.2)$ & & 64 (83.1) & & \\
\hline Grade 1 & $5(6.5)$ & $6(7.8)$ & & $9(11.7)$ & & \\
\hline Grade 2 & 0 & 0 & & $4(5.2)$ & & \\
\hline \multicolumn{7}{|l|}{ LA strain (\%) } \\
\hline$\varepsilon \mathrm{R}$ & $30.8 \pm 11.3$ & $31.3 \pm 10.2$ & .741 & $30.5 \pm 10.9$ & .601 & .822 \\
\hline$\varepsilon \mathrm{CT}$ & $13.2 \pm 4.5$ & $13.0 \pm 4.3$ & .818 & $12.5 \pm 4.5$ & .409 & .286 \\
\hline$\varepsilon C D$ & $17.7 \pm 8.7$ & $18.2 \pm 8.5$ & 561 & $18.0 \pm 8.6$ & .827 & .753 \\
\hline
\end{tabular}

Abbreviations: $\varepsilon C D=L A$ conduct strain; $\varepsilon C T=L A$ contractile strain; $\varepsilon R=L A$ reservoir strain; $B S A=$ body surface area; DecT = deceleration time; $\mathrm{LA}=$ left atrial; $\mathrm{LVEF}=$ left ventricular ejection fraction; $\mathrm{LVGLS}=$ left ventricular global longitudinal strain; $T R=$ tricuspid regurgitation.

TABLE 5 Significant changes in left atrial strain parameters

\begin{tabular}{lllll}
$\mathrm{n}(\%)$ & Reduction 1-2 & Reduction 2-3 & Reduction 1-3 & Total reduction \\
\hline$\varepsilon \mathrm{R}$ & $3(3.9)$ & $8(10.4)$ & $7(9.1)$ & $11(14.3)$ \\
$\varepsilon \mathrm{CT}$ & $8(10.4)$ & $9(11.7)$ & $8(10.4)$ & $16(20.8)$ \\
$\varepsilon \mathrm{CD}$ & $7(9.1)$ & $6(7.8)$ & $4(5.2)$ & $13(16.9)$ \\
\hline
\end{tabular}

Abbreviations: $\varepsilon C D=L A$ conduit strain; $\varepsilon C T=L A$ contractile strain; $\varepsilon R=L A$ reservoir strain. remains questionable in the oncology setting because there might be E velocity fluctuations as a consequence of changes in loading conditions associated to side effects of chemotherapy (nausea, vomiting, and diarrhea) and not as a result of real change in left ventricular diastolic performance. ${ }^{17}$ In this regard, $\mathrm{e}^{\prime}$ is more independent of preload. ${ }^{17}$ In our sample, we demonstrated a significant worsening of diastolic function during treatment. However, the main determinant of worsening was age and this is in accordance with previous observations of LV systolic cardiotoxicity being more frequent in patients older than 65 years. ${ }^{4}$ We can conclude that these agents are more detrimental in older individuals, and thus, a more strict surveillance should be undertaken in those patients for early detection of those changes and to decide on the need to use less aggressive regimen. In our sample, we also did not observe a significant relationship between diastolic dysfunction development/worsening and LV systolic dysfunction during follow-up, and for that reason, no recommendations about treatment interruption can be suggested.

Left atrial indexed volume remains the main echocardiographic parameter to assess the remodeling and indirectly the function of the LA and is a powerful prognostic tool. ${ }^{17-20}$ However, volumetric measures of LA function are limited by lower sensitivity in early disease states and can be increased due to other diseases. ${ }^{19}$ LA myocardial analysis using 2DSTE identifies LA dysfunction despite normal LA ejection fraction and normal LA indexed volume in patients with LV diastolic dysfunction. ${ }^{17}$ Thus, myocardial LA analysis using 2DSTE has several advantages over volumetric LA measurements. The advantages of left atrial strain by STE are the rapid and easy performance, angle independence, it is less affected by side lobes, reverberations and dropout artifacts, the possibility of offline processing, and provides qualitative assessment of LA function. ${ }^{17}$ The main disadvantages are the frame rate dependence, the potential errors in epicardial/endocardial border tracing and the absence of a dedicated analysis software. ${ }^{17}$ Unlike LV strain, LA strain using STE has not been validated, but there is a growing body of outcome data supporting its diagnostic and prognostic value. ${ }^{8-}$ ${ }^{14}$ Our results showed very good reproducibility with the technique.

LA strain parameters are usually higher in women, compared to men. ${ }^{21}$ With increasing age, it decreases progressively, being more pronounced in women, and independently of baseline characteristics such 


\begin{tabular}{|c|c|c|c|c|}
\hline & $\begin{array}{l}\text { Univariate OR } \\
(95 \% \mathrm{Cl})\end{array}$ & $P$-value & $\begin{array}{l}\text { Multivariate OR } \\
(95 \% \mathrm{Cl})\end{array}$ & $P$-value \\
\hline \multicolumn{5}{|l|}{ Significant $\varepsilon R$ change } \\
\hline Docetaxel & $0.21(0.06-0.84)$ & .027 & $0.26(0.06-1.10)$ & .067 \\
\hline Diastolic dysfunction & $3.20(0.79-12.98)$ & .100 & $2.19(0.45-9.89)$ & .307 \\
\hline \multicolumn{5}{|l|}{ Significant $\varepsilon C D$ change } \\
\hline Hypertension & $3.81(1.11-13.10)$ & .034 & $2.34(0.51-10.82)$ & .277 \\
\hline Docetaxel & $0.30(0.08-1.09)$ & .068 & $0.40(0.10-1.64)$ & .201 \\
\hline Anastrazol & $4.30(1.01-18.26)$ & .048 & $2.78(0.55-13.94)$ & .213 \\
\hline Diastolic dysfunction & $3.82(1.02-14.31)$ & .047 & $1.34(0.23-7.68)$ & .741 \\
\hline \multicolumn{5}{|l|}{ Significant $\varepsilon C T$ change } \\
\hline Age (per 10-y increase) & $0.55(0.32-0.95)$ & .031 & $0.51(0.28-0.91)$ & .023 \\
\hline Baseline LVEF & $1.08(0.99-1.18)$ & .066 & $1.11(1.00-1.23)$ & .055 \\
\hline Pertuzumab & $3.06(0.91-10.34)$ & .072 & $2.31(0.49-10.85)$ & .287 \\
\hline Neoadjuvant chemotherapy & $3.94(1.25-12.41)$ & .019 & $2.68(0.66-10.95)$ & .170 \\
\hline
\end{tabular}

TAB LE 6 Factors associated with left atrial strain parameters significant changes

Abbreviations: $\varepsilon C D=$ left atrial conduit strain; $\varepsilon C T=$ left atrial contractile strain; $\varepsilon R=$ left atrial reservoir strain; $\mathrm{Cl}$ = confidence interval; $\mathrm{LVEF}=$ left ventricular ejection fraction; $\mathrm{OR}=$ odds ratio.

as blood pressure, LA volume or LV diastolic function. ${ }^{17-21}$ With increasing age, impaired LV relaxation reduces passive atrial conduit function and LA stiffness decreases reservoir function, limiting LV filling. ${ }^{21}$ With senescence, LA contraction augments as a compensatory mechanism with a late decrease with further aging. ${ }^{21}$ The same changes are reported with LV diastolic dysfunction. ${ }^{22,23}$ In early stages, LA reservoir and conduit strain decreases and an increase in its booster function can compensate filling. But with prolonged dysfunction, LA dilation occurs, and eventually, LA mechanical function shows a fall in the relative contribution of LA pump to LV filling. This is particularly important for heart failure with preserved ejection fraction progression because LA strain parameters are independently associated with abnormal exercise hemodynamics. ${ }^{24}$ Some authors suggest the use of LA reservoir strain (peak LA strain) as a promising prognostic marker in heart failure. ${ }^{25}$

Our baseline values for all strain parameters, with the exception of $\varepsilon C D$, were lower in comparison with the normal values reported in the literature. ${ }^{19}$ Our explanation is the difference in baseline characteristics of our sample in comparison with the healthy subjects analyzed in the meta-analysis that defined normal LA strain values. Reference values for LA strain were defined for patients without cardiovascular risk factors. Our sample has several patients with an age $>60$ years, hypertension and baseline diastolic dysfunction that was not present in the previous meta-analysis.

We observed a substantial reduction in LA contractile function in more than $20 \%$ of the patients, although all other parameters were also changes but in fewer patients. The reduction in contractile function was only independently associated with age, being in that case protective. As previously mentioned, age is one of the most important predictors of LA dysfunction, independently of other characteristics such as LV diastolic dysfunction and as such, our results are in accordance with previous reports. Thus, the most likely explanation is that, as previously mentioned, $\varepsilon \mathrm{R}$ and $\varepsilon \mathrm{CD}$ usually deteriorate with increasing age, but contractile function is positively correlated with age (increases with age). ${ }^{19,21}$ Augmented LA active contraction occurs as a compensatory mechanism with senescence. ${ }^{19,21}$ For that reason, in older patients, we expect a less significant or delayed decrease in LA contractile function by strain analysis, justifying the inverse independent association observed.

\section{1 | Limitations}

Although our sample size is adequate according to our sample size analysis, it is, nonetheless, relatively small. For that reason, our results require confirmation in larger samples.

This is a retrospective study, and strain measurements were not a prespecified test in routine evaluation. Thus, a significant number of patients did not have adequate images for strain analysis. Our feasibility was $77 \%$, a little lower compared to other studies that report exclusion rates around $15 \%$ due to poor image quality. In our case, the main reason was inadequate storing of images and not image quality.

Intervendor variability is a problem previously reported in strain analysis with speckle tracking. However, in the previously mentioned meta-analysis for the LA strain reference values, they found no significant differences between vendors. ${ }^{19}$

Another potential limitation of left atrial strain is LA wall thinness, with the possibility of wall dropout and poor tissue tracking.

\section{5 | CONCLUSION}

In our sample of women with breast cancer, during the first year of breast cancer treatment, there is a significant worsening in diastolic function, being age the only independent predictor. Neither baseline diastolic dysfunction nor LV systolic dysfunction had any 
significant impact on LV diastolic dysfunction. Regarding LA function, the contractile function was the one that was more frequently compromised, being age the only independent predictor, but as a protective factor.

\section{CONFLICT OF INTERESTS}

None declared.

\section{ORCID}

Ana Teresa Timóteo (iD https://orcid.org/0000-0001-6061-9663

\section{REFERENCES}

1. World Health Organization. The Global Cancer Observatory. Available from: gco.iarc-fr/total/data/factsheets/cancer/20Breast-fact-sheet.pdf

2. GBD 2016 Causes of Death Collaborators. Global, regional, and national age-sex-specific mortality for 269 causes of death, 1980-2016: a systematic analysis for the Global Burden of Disease Study 2016. Lancet. 2017;390:1151-1210.

3. General Directorate of Health. Programa Nacional para doenças oncológicas. Available from: www.dgs.pt

4. Zamorano JL, Lancelloti P, Asteggiano R, et al. 2016 ESC position paper on cancer treatments and cardiovascular toxicity developed under the auspices of the ESC committee for practice guidelines. Eur Heart J. 2016;37:2768-2801.

5. Eschenhagen T, Force T, Ewer M, et al. Cardiovascular side effects of cancer therapies: a position statement from the Heart Failure Association of the European Society of Cardiology. Eur J Heart Fail. 2011;13:1-10.

6. Portugal G, Moura Branco L, Galrinho A, et al. Global and regional patterns of longitudinal strain in screening for chemotherapy-induced cardiotoxicity. Rev Port Cardiol. 2017;36:9-15.

7. Badano LP, Kolias TJ, Muraru D, et al. Standardization of left atrial, right ventricular, and right atrial deformation imaging using twodimensional speckle tracking echocardiography: a consensus document of the EACVI/ASE/Industry Task Force to standardize deformation imaging. Eur Heart J Cardiovasc Imaging. 2018;19:591-600.

8. Cameli M, Ciccione MM, Maiello M, et al. Speckle-tracking analysis: a new tool for left atrial function analysis in systemic hypertension: an overview. J Cardiovasc Med. 2016;17:339-343.

9. Mondillo S, Cameli M, Caputo NL. Early detection of left atrial strain abnormalities by speckle tracking in hypertensive and diabetic patients with normal left atrial size. J Am Soc Echocardiogr. 2011;24:898-908.

10. Malagoli A, Rossi L, Bursi F, et al. Left atrial function predicts cardiovascular events in patients with chronic heart failure with reduced ejection fraction. J Am Soc Echocardiogr. 2019;32:248-256.

11. Antoni ML, Ten Brinke EA, Marsan MA. Comprehensive assessment of changes in left atrial volumes and function after ST-segment elevation acute myocardial infarction: role of two-dimensional speckle tracking strain imaging. J Am Soc Echocardiogr. 2011;24:1126-1133.

12. Todaro MC, Carerg S, Khandheria B, et al. Usefulness of atrial function for risk stratification in asymptomatic severe aortic stenosis. J Cardiol. 2016;67:71-79.

13. Mochizuki A, Yuda S, Oi Y, et al. Assessment of left atrial deformation and synchrony by three-dimensional speckle-tracking echocardiography: comparative studies in healthy subjects and patients with atrial fibrillation. J Am Soc Echocardiogr. 2013;26:165-174.
14. Obokata M, Negishi K, Kurosava K, et al. Left atrial strain provides incremental value for embolism risk stratification over $\mathrm{CHA}(2)$ DS(2)-VASc score and indicates prognostic impact in patients with atrial fibrillation. J Am Soc Echocardiogr. 2014;27:709-716.

15. Lang RM, Badano LP, Mor-Avi V, et al. Recommendations for cardiac chamber quantification by echocardiography in adults: an update from the American Society of Echocardiography and the European Association of Cardiovascular Imaging. Eur Heart J Cardiovasc Imaging. 2015;16:233-271.

16. Nagueh SF, Smiseth $\mathrm{OA}$, Appleton $\mathrm{CP}$, et al. Recommendations for the evaluation of left ventricular diastolic function by echocardiography: an update from the American Society of Echocardiography and the European Association of Cardiovascular Imaging. Eur Heart J Cardiovasc Imaging. 2016;17:1321-1360.

17. Cameli M, Mandoli GE, Loiacono F, et al. Left atrial strain: a new parameter for assessment of left ventricular filling pressure. Heart Fail Rev. 2016;21:65-76.

18. Morris DA, Takeuchi M, Krisper M, et al. Normal values and clinical relevance of left atrial myocardial function analyzed by speckle-tracking echocardiography: multicenter study. Eur Heart J Cardiovasc Imaging. 2015;16:364-372.

19. Pathan F, D'Elia N, Nolan M, et al. Normal ranges of left atrial strain by speckle-tracking echocardiography: a systematic review and meta-analysis. J Am Soc Echocardiogr. 2017;30:59-70.

20. Meel R, Khandheria BK, Peters F, et al. Effects of age on left atrial volume and strain parameters using echocardiography in a normal black population. Echo Res Pract. 2016;3:115-123.

21. Liao JN, Chao TF, Kuo JY, et al. Age, sex, and blood pressure-related influences on reference values of left atrial deformation and mechanics from a large scale Asian population. Circ Cardiovasc Imaging. 2017;10:e006077. https://doi.org/10.1161/CIRCI MAGING.116.006077

22. Saikhan LA, Hughes AD, Chung WS, et al. Left atrial function in heart failure with mid-range ejection fraction differes from that of heart failure with preserved ejection fraction: a 2D speckletracking echocardiographic study. Eur Heart J Cardiovasc Imaging. 2019;20:279-290.

23. Reddy Y, Obokata M, Egbe A, et al. Left atrial strain and compliance in the diagnostic evaluation of heart failure with preserved ejection fraction. Eur J Heart Fail. 2019;21:891-900.

24. Telles F, Nanayakkara S, Evans S, et al. Impaired left atrial strain predicts abnormal exercise haemodynamics in heart failure with preserved ejection fraction. Eur J Heart Fail. 2019;21:495-505.

25. Thomas L, Marwick TH, Popescu BA, et al. Left atrial structure and function, and left ventricular diastolic dysfunction. J Am Coll Cardiol. 2019;73:1961-1977.

\section{SUPPORTING INFORMATION}

Additional supporting information may be found online in the Supporting Information section at the end of the article.

Table S1. Reproducibility analysis for left atrial strain by 2-dimensional speckle tracking echocardiography.

How to cite this article: Timóteo AT, Moura Branco L, Filipe F, et al. Cardiotoxicity in breast cancer treatment: What about left ventricular diastolic function and left atrial function?. Echocardiography. 2019;36:1806-1813. https://doi. org/10.1111/echo.14487 